\title{
Damage to the Insula Disrupts Addiction to Cigarette Smoking
}

\author{
Nasir H. Naqvi ${ }^{1}$, David Rudrauf ${ }^{1,2}$, Hanna Damasio ${ }^{3,4}$, and Antoine Bechara $1,3,4,{ }^{*}$ \\ ${ }^{1}$ Division of Cognitive Neuroscience, Department of Neurology, University of lowa Carver College \\ of Medicine, 200 Hawkins Drive, lowa City, IA 52242, USA \\ ${ }^{2}$ Laboratory of Computational Neuroimaging, Department of Neurology, University of lowa Carver \\ College of Medicine, 200 Hawkins Drive, lowa City, IA 52242, USA \\ ${ }^{3}$ Dornsife Cognitive Neuroscience Imaging Center, SGM 501, University of Southern California, \\ Los Angeles, CA 90089, USA \\ ${ }^{4}$ Brain and Creativity Institute, HNB B26, University of Southern California, Los Angeles, CA \\ 90089, USA
}

\begin{abstract}
A number of brain systems have been implicated in addictive behavior, but none have yet been shown to be necessary for maintaining the addiction to cigarette smoking. We found that smokers with brain damage involving the insula, a region implicated in conscious urges, were more likely than smokers with brain damage not involving the insula to undergo a disruption of smoking addiction, characterized by the ability to quit smoking easily, immediately, without relapse, and without persistence of the urge to smoke. This result suggests that the insula is a critical neural substrate in the addiction to smoking.
\end{abstract}

Cigarette smoking, the most common preventable cause of morbidity and mortality in the developed world (1), is an addictive behavior. Despite being aware of negative consequences, many smokers have difficulty quitting, and even those who quit experience urges to smoke and tend to relapse $(2,3)$. These phenomena appear to arise from long-term adaptations within specific neural systems. Subcortical regions, such as the amygdala, the nucleus accumbens, and the mesotelencephalic dopamine system, have been shown in animal models to promote the self-administration of drugs of abuse $(4,5)$. Functional imaging studies have shown that exposure to drug-associated cues activates cortical regions such as the anterior cingulate cortex, the orbitofrontal cortex, and the insula (6-13). Among these regions, the insula is of particular interest because of its potential role in conscious urges. The insula has been proposed to function in conscious emotional feelings through its role in the representation of bodily (interoceptive) states (14-16). Activity within the insula on both sides of the brain has been shown to correlate with subjective cue-induced drug urges $(7,8,11)$. It has also been shown that a high amount of activity in the right insula during a simple decision-making task is associated with relapse to drug use (17). Given its potential role in cognitive and emotional processes that promote drug use, the question arises as to whether the insula is necessary for maintaining addiction to smoking. We

\footnotetext{
*To whom correspondence should be addressed: bechara@usc.edu.

Supporting Online Material

www.sciencemag.org/cgi/content/full/315/5811/531/DC1

Materials and Methods

Figs. S1 and S2

Tables S1 to S5

References
} 
hypothesized that the insula is a critical neural substrate in the addiction to smoking. We predicted, therefore, that damage to the insula would disrupt addiction to smoking.

We identified 19 cigarette smokers who had acquired brain damage that included the insula (18). Six of these patients had right insula damage, and 13 had left insula damage. We also identified a group of 50 cigarette smokers who had acquired damage that did not include the insula. All of these patients had been smoking more than five cigarettes per day for more than 2 years at the time of lesion onset. The groups were matched with respect to several characteristics, including the number of cigarettes they were smoking at lesion onset, the total number of years they had been smoking at lesion onset, and the etiology of their brain damage (Fig. 1 and table S1).

First, we performed a logistic regression analysis in which the dependent variable was whether or not patients quit smoking some time after lesion onset (i.e., whether or not they were smoking at the time of the study). The independent variable of interest was the extent of damage in the insula on a given side. An estimate of the total extent of the lesion was entered as a nuisance covariable (Materials and Methods). We found that the likelihood of quitting smoking after a lesion in either the right or the left insula was not significantly higher than the likelihood of quitting after a noninsula lesion (odds ratio $=2.94, \chi^{2}=2.74$, and $P=0.10$ ). When we examined the right and left insulae separately, we found that the likelihood of quitting smoking was not significantly higher after a right insula lesion than after a noninsula lesion (odds ratio $=2.53, \chi^{2}=2.98$, and $P=0.08$ ), nor was it significantly higher after a left insula lesion compared with after a noninsula lesion (odds ratio $=1.44, \chi^{2}$ $=1.12$, and $P=0.29$ ) (Fig. 2 and table S3). One explanation of this null finding is that, whereas the insula-lesioned patients may have quit smoking due to a disruption of smoking addiction, the noninsula-lesioned patients may have quit smoking at a similar rate because they were concerned about the negative consequences of smoking. Simply determining whether the patients were smoking at the time of the study did not address this distinction.

To more specifically assess a disruption of smoking addiction, we asked all of the patients who quit smoking after lesion onset a set of questions aimed at their recollection of the experience of quitting. Patients were classified as having had a disruption of smoking addiction if they fulfilled all four of the following criteria: (i) reporting quitting smoking less than 1 day after lesion onset, (ii) reporting that they did not start smoking again after they quit, (iii) rating the difficulty of quitting as less than three on a scale of one to seven, and (iv) reporting feeling no urges to smoke since quitting. According to these criteria, 16 of the patients who quit smoking after lesion onset were classified as having a disruption of smoking addiction. The 16 quitters who failed to meet all four of these criteria, along with all 37 nonquitters, were considered to have no disruption of smoking addiction (Fig. 2).

We performed a logistic regression in which the dependent variable was whether or not patients underwent a disruption of smoking addiction after lesion onset as defined by the above criteria. As before, the independent variable of interest was the extent of damage to the insula on a given side, whereas the estimate of the total extent of the lesion was entered as a nuisance covariable. We found that the likelihood of having a disruption of smoking addiction after a lesion in either the right or the left insula was significantly higher than the likelihood of having a disruption of smoking addiction after a noninsula lesion (odds ratio $=$ $22.05, \chi^{2}=16.64$, and $P=0.0005$ ). When we examined the right and left insulae separately, we found that the likelihood of having a disruption of smoking addiction was significantly higher after a right insula lesion than after a noninsula lesion (odds ratio $=10.87, \chi^{2}=$ 12.90 , and $P=0.0003$ ) and was also significantly higher after a left insula lesion compared with after a noninsula lesion (odds ratio $=3.61, \chi^{2}=10.33$, and $P=0.001$ ) (Fig. 2 and table S3). Although it appears that effects may be somewhat larger with right insula lesions 
compared with left insula lesions, the sample sizes were too small to confirm this statistically.

We then conducted a similar logistic regression that included only the patients in our sample who quit smoking after lesion onset (thus, we were not required to assume that patients who continued to smoke after lesion onset had an intact smoking addiction). We found that five of five of the patients who quit smoking after a right insula lesion and seven of eight of the patients who quit smoking after a left insula lesion met the criteria for having a disruption of smoking addiction, compared to 4 of 19 of the patients who quit smoking after a noninsula lesion (right insula-lesioned patients versus noninsula-lesioned patients: odds ratio $=6.55$, $\chi^{2}=7.76$, and $P=0.005$; left insula-lesioned patients versus noninsula-lesioned patients: odds ratio $=7.19, \chi^{2}=10.06$, and $P=0.002$ ). Putting the right and left sides together, 12 of 13 patients who quit smoking after a lesion in the insula did so with a disruption of smoking addiction. Relative to noninsula-lesioned patients, this translates into an odds ratio of 136.49 as estimated by the logistic regression $\left(\chi^{2}=15.48\right.$ and $\left.P=0.00008\right)$ (Fig. 2 and table S3).

In our sample, the patients with insula lesions tended also to have damage in adjacent areas (Fig. 1). This raises the question of whether the observed effects were necessarily due to insula damage or whether they required damage in one or more areas adjacent to the insula. To address this issue, we performed a region-by-region logistic regression analysis that estimated, for each region of the brain that we sampled, the likelihood of having a disruption of smoking addiction after a lesion that included the region compared to a lesion that did not include the region. This analysis included all of the patients in the sample. We found that the only regions in which lesions were significantly associated with an increased likelihood of having a disruption of smoking addiction were the right and left insulae (Fig. 3). On the left side, there were near-significant effects in regions adjacent to the insula, such as the putamen. We cannot rule out the possibility that some of these regions independently or cumulatively play a role in smoking addiction. For example, evidence from animal studies suggests that the dorsal striatum, which includes the putamen, is involved in learning and expression of drug-use habits (4). However, for most of these regions the patients with lesions who had a disruption of smoking addiction also had damage in the insula (table S4), suggesting that apparent effects of lesions in these regions were due to a bystander effect. We did find four patients who had a disruption of smoking addiction after suffering from brain damage that did not involve the insula. When we examined their lesions, we found that each of them had damage in a unique set of regions (table S5). This raises the possibility that certain patients may undergo a disruption of smoking addiction as a general effect of suffering from a brain injury.

The results indicate that smokers who acquire insula damage are very likely to quit smoking easily and immediately and to remain abstinent. In addition, smokers with insula damage are very likely to no longer experience conscious urges to smoke after quitting. These findings are consistent with previous functional imaging evidence showing that activity in the insula is correlated with subjective drug urges $(7,8,11)$. Additionally, the results provide evidence that subjective urges are an important factor in maintaining smoking addiction. However, urges may not be the only factor that promotes smoking. Recent theories of addiction propose that usual drug use in addicted individuals is driven primarily by automatic or implicit motivational processes, such as habits (4) and incentive salience wanting (19). Conscious urges come into play when there is an impediment to drug use, such as an attempt to quit or to resist relapse (20). The present results are consistent with this view. However, it remains to be seen whether insula damage spares the automatic tendency to smoke. It also remains to be seen whether patients with insula damage still obtain pleasure from smoking, because pleasure and urge may be dissociable facets of smoking reward (19). 
Our sample included a number of patients with damage to the orbitofrontal cortex (Fig. 1), a region that, like the insula, has been implicated by functional imaging studies to play a role in conscious drug urges $(6,8,9,11-13)$. We found no association between lesions in the orbitofrontal cortex and a disruption of smoking addiction (Fig. 3 and table S4). One explanation of this result is that smokers who acquire orbitofrontal damage experience a reduction in conscious urges but continue to smoke because their automatic tendency to smoke is still intact. At the same time, these patients may have a low likelihood of attempting to quit smoking after suffering from a brain injury, because the orbitofrontal region is critical for decisions that override the automatic tendency to obtain immediate rewards in order to avoid future negative consequences $(21,22)$. Insula-lesioned patients, in contrast, may not have such severe decision-making deficits and thus may be likely to attempt to quit smoking after suffering from a brain injury.

The results of this study suggest that the insula is a critical neural substrate for the urge to smoke, although they do not in themselves indicate why the insula, a region known to play a role in the representation of the bodily states (16), would play such an important role in urge. A clue may be provided by the account of one patient in our sample who quit smoking immediately after he suffered a stroke that damaged his left insula. He stated that he quit because his "body forgot the urge to smoke" (23). His experience suggests that the insula plays a role in the feeling that smoking is a bodily need. Indeed, much of the pleasure and satiety that is obtained from smoking is derived from its bodily effects, in particular its impact on the airway $(24,25)$. In addition, nicotine withdrawal is associated with changes in autonomic and endocrine function $(26,27)$, which may contribute to its unpleasantness. Current evidence suggests that the insula plays a role in conscious feelings by anticipating the bodily effects of emotional events $(14,15)$. The insula may therefore function in the conscious urge to smoke by anticipating pleasure from the airway effects of smoking and/or relief from the aversive autonomic effects of nicotine withdrawal. Thus, damage to the insula could lead a smoker to feel that his or her body has "forgotten" the urge to smoke.

An important question pertains to whether insula lesions cause a disruption of motivated behaviors other than smoking. In a follow-up survey, we found that none of the patients with insula damage who had a disruption of smoking addiction admitted to any reductions in their pleasure from eating, their desire to eat, or their intake of food. This does not preclude the possibility that these patients had some impairment of taste perception $(28,29)$ or had deficits in other motivated behaviors that we did not assess. One possibility is that motivated behaviors that are fundamental to survival, such as eating, are supported by redundant neural mechanisms that are difficult to disrupt with a lesion in a single brain region. A related possibility is that the insula is critical for behaviors whose bodily effects become pleasurable through learning; although the bodily effects of eating are inherently pleasurable, the bodily effects of smoking are initially aversive and become pleasurable as addiction develops (25). It would be interesting to see how insula damage affects other learned pleasures.

Our findings suggest that therapies that modulate the function of the insula will be useful in helping smokers quit. For example, sensory replacements for smoking, such as denicotinized cigarettes and irritant inhalers, are highly effective in reducing urges and promoting abstinence $(30,31)$. Such therapies may work by engaging sensory representations of the airway within the insula, thereby satisfying the "bodily need" to smoke. Future pharmacologic therapies may target neurotransmitter receptors that are expressed within the insula. In addition, the efficacy of various smoking cessation therapies may be monitored by measuring activity within the insula with functional brain imaging. Lastly, the findings of this study demonstrate that conscious feelings, such as urges, are an important component of addiction. 


\section{Supplementary Material}

Refer to Web version on PubMed Central for supplementary material.

\section{Acknowledgments}

The authors thank A. Damasio, T. Grabowski, D. Tranel, and B. Porter for helpful comments on the manuscript and S. Mehta for expert advice on the logistic regression analyses. This research was supported by National Institute on Drug Abuse grants F30 DA016847 (N.H.N.) and R21 DA16708 (A.B.) and National Institute of Neurological Disorders and Stroke grant P01 NS019632 (A.B. and H.D.).

\section{References and Notes}

1. Peto R, Lopez AD, Boreham J, Thun M, Heath C Jr. Lancet. 1992; 339:1268. [PubMed: 1349675]

2. American Psychiatric Association (A.P.A.). Diagnostic and Statistical Manual of Mental Disorders Text Revision: DSM-IV-TR. 4. A.P.A; Washington, DC: 2000. p. 191-296.

3. U.S. Department of Health and Human Services. 1988 Surgeon General's Report: The Health Consequences of Smoking: Nicotine Addiction. Vol. chap 6. U.S. Government Printing Office; Rockville, MD: 1988. p. 377-458.

4. Everitt BJ, Robbins TW. Nat Neurosci. 2005; 8:1481. [PubMed: 16251991]

5. Pontieri FE, Tanda G, Orzi F, Di Chiara G. Nature. 1996; 382:255. [PubMed: 8717040]

6. Grant S, et al. Proc Natl Acad Sci USA. 1996; 93:12040. [PubMed: 8876259]

7. Bonson KR, et al. Neuropsychopharmacology. 2002; 26:376. [PubMed: 11850152]

8. Brody AL, et al. Arch Gen Psychiatry. 2002; 59:1162. [PubMed: 12470133]

9. Goldstein RZ, Volkow ND. Am J Psychiatry. 2002; 159:1642. [PubMed: 12359667]

10. Garavan H, et al. Am J Psychiatry. 2000; 157:1789. [PubMed: 11058476]

11. Wang GJ, et al. Life Sci. 1999; 64:775. [PubMed: 10075110]

12. Sell LA, et al. Drug Alcohol Depend. 2000; 60:207. [PubMed: 10940548]

13. Myrick H, et al. Neuropsychopharmacology. 2004; 29:393. [PubMed: 14679386]

14. Damasio AR, et al. Nat Neurosci. 2000; 3:1049. [PubMed: 11017179]

15. Damasio, AR. The Feeling of What Happens: Body and Emotion in the Making of Consciousness. Harcourt; Chicago: 2000.

16. Craig AD. Nat Rev Neurosci. 2002; 3:655. [PubMed: 12154366]

17. Paulus MP, Tapert SF, Schuckit MA. Arch Gen Psychiatry. 2005; 62:761. [PubMed: 15997017]

18. All of the patients in this study were selected from the patient registry of the Division of

Behavioral Neurology and Cognitive Neuroscience, Department of Neurology, University of Iowa. Selection criteria are detailed in the Materials and Methods. The insula was defined here as the region bounded anteriorly by the limen insulae and posteriorly, inferiorly, and superiorly by the circular insular sulcus. This included both anterior and posterior insular cortices and underlying white matter.

19. Berridge KC, Robinson TE. Trends Neurosci. 2003; 26:507. [PubMed: 12948663]

20. Tiffany ST. Psychol Rev. 1990; 97:147. [PubMed: 2186423]

21. Bechara A. Nat Neurosci. 2005; 8:1458. [PubMed: 16251988]

22. Bechara A, Tranel D, Damasio H. Brain. 2000; 123:2189. [PubMed: 11050020]

23. Patient N. is a right-handed male who was 38 years old when we interviewed him. N. started smoking at the age of 14. At the time of his stroke, he was smoking more than 40 unfiltered cigarettes per day and was enjoying smoking very much. By his own admission, he was heavily addicted to smoking. He recalled that he used to experience frequent urges to smoke, especially upon waking, after eating, when he drank coffee or alcohol, and when he was around other people who were smoking. He often found it difficult to refrain from smoking in situations where it was inappropriate, e.g., at work or when he was sick and bedridden. He was aware of the health risks of smoking before his stroke but was not particularly concerned about those risks. Before his stroke, he had never tried to stop smoking, and he had had no intention of doing so. N. smoked his 
last cigarette on the evening before his stroke. When asked about his reason for quitting smoking, he stated simply, "I forgot that I was a smoker." When asked to elaborate, he said that he did not forget the fact that he was a smoker but rather that "my body forgot the urge to smoke." He felt no urge to smoke during his hospital stay, even though he had the opportunity to go outside to smoke. His wife was surprised by the fact that he did not want to smoke in the hospital, given the degree of his prior addiction. N. recalled how his roommate in the hospital would frequently go outside to smoke and that he was so disgusted by the smell upon his roommate's return that he asked to change rooms. He volunteered that smoking in his dreams, which used to be pleasurable before his stroke, was now disgusting. N. stated that, although he ultimately came to believe that his stroke was caused in some way by smoking, suffering a stroke was not the reason why he quit. In fact, he did not recall ever making any effort to stop smoking. Instead, it seemed to him that he had spontaneously lost all interest in smoking. When asked whether his stroke might have destroyed some part of his brain (fig. S2) that made him want to smoke, he agreed that this was likely to have been the case.

24. Naqvi NH, Bechara A. Pharmacol Biochem Behav. 2005; 81:821. [PubMed: 15996724]

25. Rose JE. Psychopharmacol Ser (Berlin). 2006; 184:274.

26. Hatsukami D, Hughes JR, Pickens R. Natl Inst Drug Abuse Res Monogr. 1985; 53:56.

27. Pickworth WB, Fant RV. Psychoneuroendocrinology. 1998; 23:131. [PubMed: 9621394]

28. Pritchard TC, Macaluso DA, Eslinger PJ. Behav Neurosci. 1999; 113:663. [PubMed: 10495075]

29. Cereda C, Ghika J, Maeder P, Bogousslavsky J. Neurology. 2002; 59:1950. [PubMed: 12499489]

30. Buchhalter AR, Acosta MC, Evans SE, Breland AB, Eissenberg T. Addiction. 2005; 100:550. [PubMed: 15784070]

31. Westman EC, Behm FM, Rose JE. Chest. 1995; 107:1358. [PubMed: 7750331] 
All

$(\mathrm{N}=69)$
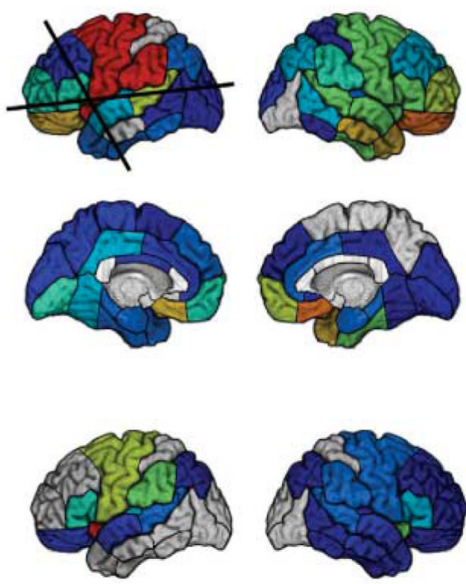

Insula

( $\mathrm{N}=19$ )
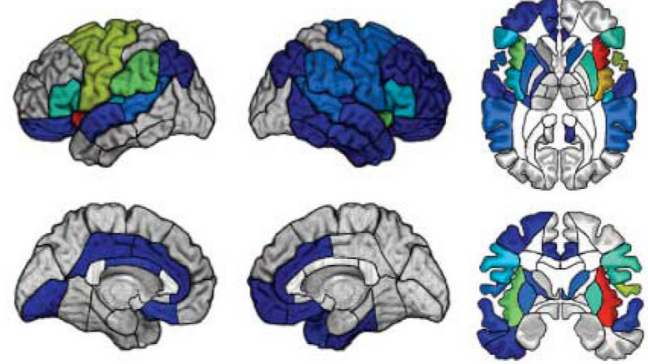

Non-

insula $(\mathrm{N}=50)$
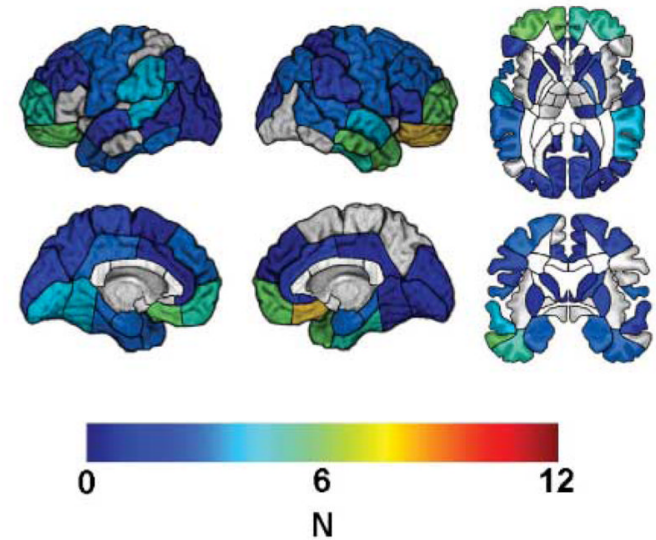

Fig. 1.

Number $(\mathrm{N})$ of patients with lesion in each of the regions identified in this study, mapped onto a reference brain. Boundaries of anatomically defined regions are drawn on the brain surface. Regions names are provided in the Materials and Methods. Regions not assigned a color contained no lesions. (Top) All patients. The horizontal line marks the transverse section of the brain shown in the top row. The vertical line marks the coronal section shown in the bottom row. (Middle) Patients with lesions that involved the insula. (Bottom) Patients with lesions that did not involve the insula. 
A

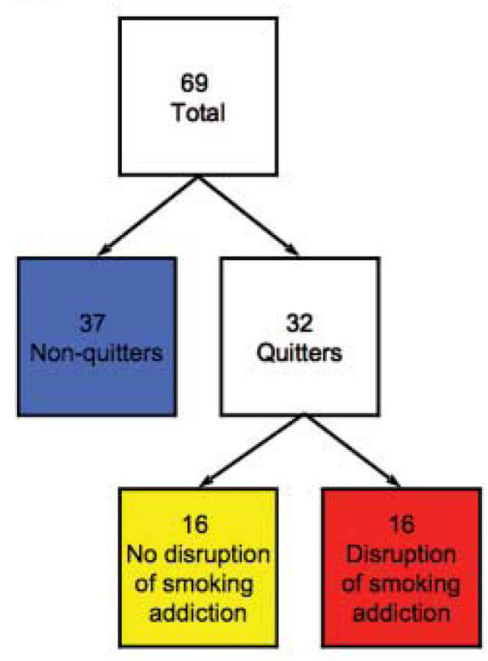

B

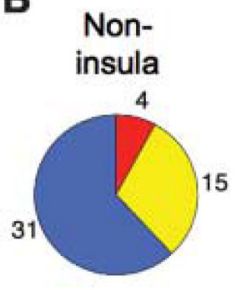

Left

insula

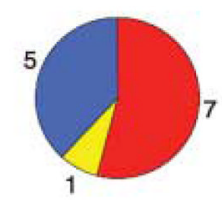

Total

insula

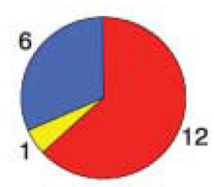

Right

insula

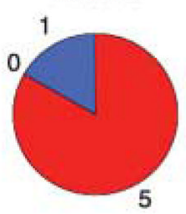

on-quitters

$\square$ Quitters with no disruption of smoking addiction

$\square$ Quitters with disruption of smoking addiction

Fig. 2.

Patients who quit smoking after lesion onset and patients who underwent a disruption of smoking addiction after lesion onset. (A) Tree diagram showing the behavioral classification of patients. (B) Pie charts illustrating the proportion of patients in each anatomical group who fell into each of the behavioral categories. The colors correspond to the behavioral group depicted in (A). These actual proportions are shown in the Materials and Methods. The proportion of patients with a disruption of smoking addiction was higher among both left insula-lesioned patients and right insula-lesioned patients compared with among noninsula-lesioned patients. 

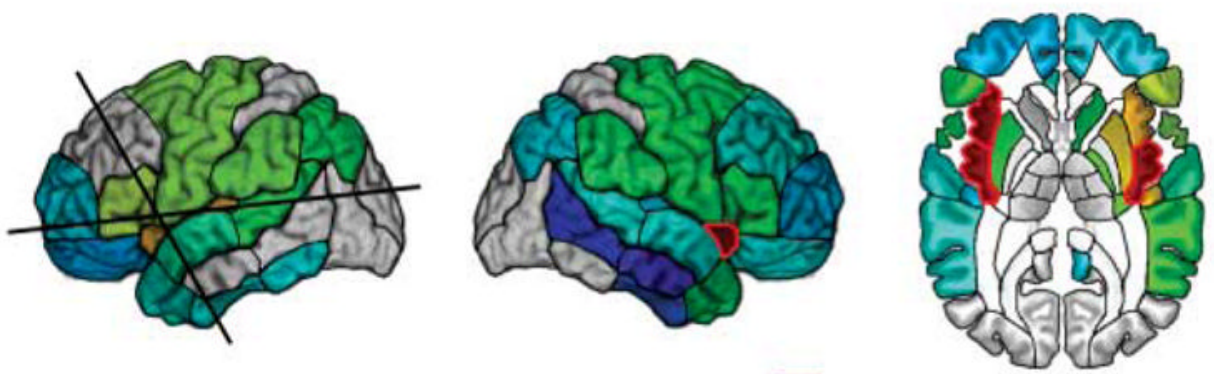

$p<0.05$
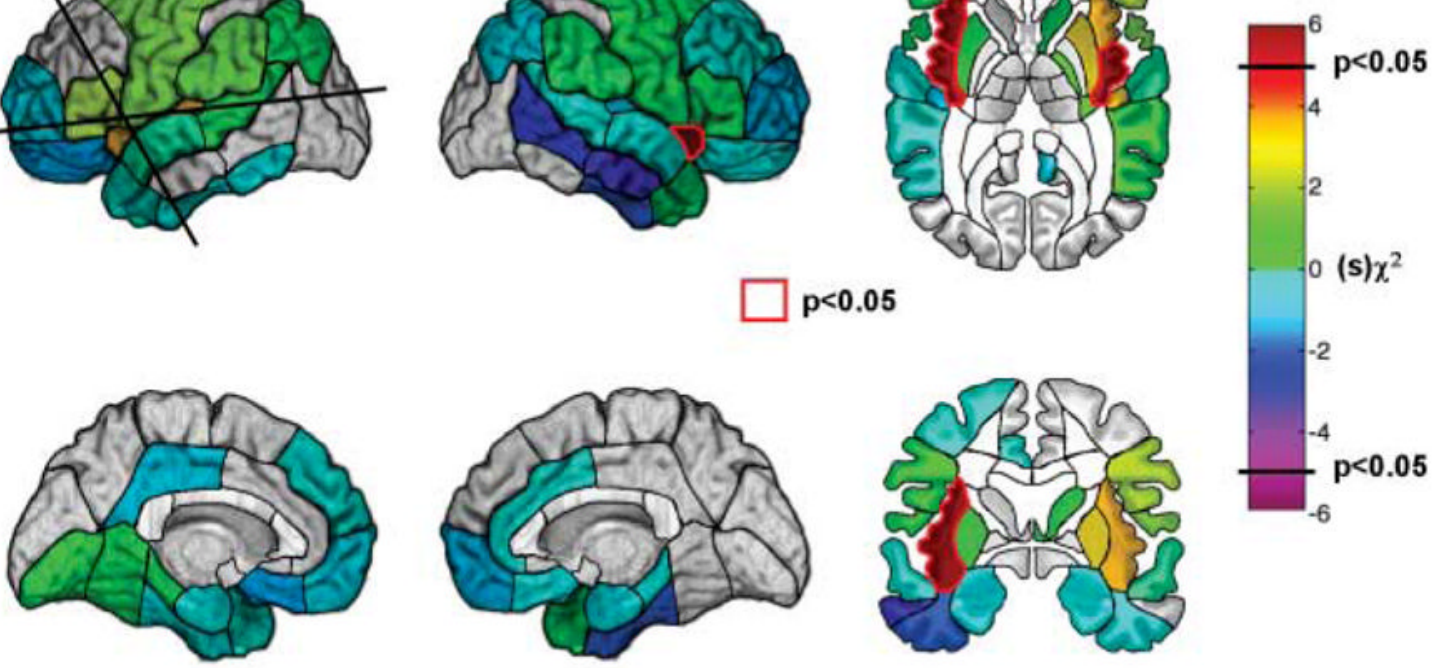

Fig. 3.

Whole-brain region-by-region logistic regression analysis. The color of each region corresponds to a $\chi^{2}$ statistic given the sign of regression coefficient obtained from the logistic regression analysis. The only regions that were assigned a color were those for which the number of patients was sufficient to detect a statistically significant effect (Materials and Methods). Regions for which there was a statistically significant association between a lesion and a disruption of smoking addiction $(P<0.05$, uncorrected) are highlighted in red. The insula is the only region on either side of the brain where a lesion was significantly associated with a disruption of smoking addiction. There were nonsignificant effects in regions on the left side that are adjacent to the insula; however, patients with damage in these regions also tended to have damage in the insula (Materials and Methods). The likelihood of having a disruption of smoking addiction was not increased after damage in the orbitofrontal cortex. 